\title{
LIVER
}

\section{Does angiogenesis have a role in the resolution of liver fibrosis?}

Angiogenesis and sinusoidal remodelling are typical features of liver fibrosis; however, the angiogenic cytokine vascular endothelial growth factor (VEGF) might also have a role in resolution of fibrosis, although this idea remains controversial and has not been investigated in many studies. "We set out to analyse the role of myeloid-cell-driven angiogenesis and vascular remodelling in the resolution of fibrosis," reports Christian Stockmann, corresponding author of a recent study published in Hepatology.

First, the researchers demonstrated that in human livers the fibrotic scar tissue has low VEGF expression, as expected. They then used established mouse models of liver fibrosis and used the targeted deletion of $V E G F$ to further investigate its role in the development and resolution of fibrosis. Interestingly, in the carbon tetrachloride $\left(\mathrm{CCL}_{4}\right)$-induced mouse model of reversible liver fibrosis, wild-type mice were able to make a full recovery whereas mice with the deletion of VEGF showed persistent fibrotic scars upon discontinuation of $\mathrm{CCL}_{4}$ treatment. Moreover, the team demonstrated that the absence of VEGF expression in myeloid cells prevented the increase of hepatic VEGF levels that seems to be necessary for resolving liver fibrosis.

Resolution of liver fibrosis requires breakdown of the extracellular matrix, which is regulated by various proteases-particularly MMPs (matrix metalloproteases) and TIMPs (tissue inhibitor of metalloproteinases). In $\mathrm{CCL}_{4}{ }^{-}$ induced mice lacking VEGF expression, MMP-2 and MMP-14 expression in endothelial cells was markedly reduced and levels of TIMP-1 and TIMP-2 were increased compared with wild-type mice.

Finally, the team were able to show that reintroduction of myeloid-cell-derived VEGF in mutant mice during recovery restored the resolution of fibrosis.

"Angiogenesis driven by myeloid-cellderived VEGF is a favourable event that contributes to the resolution of liver fibrosis," concludes Stockmann. "Based on

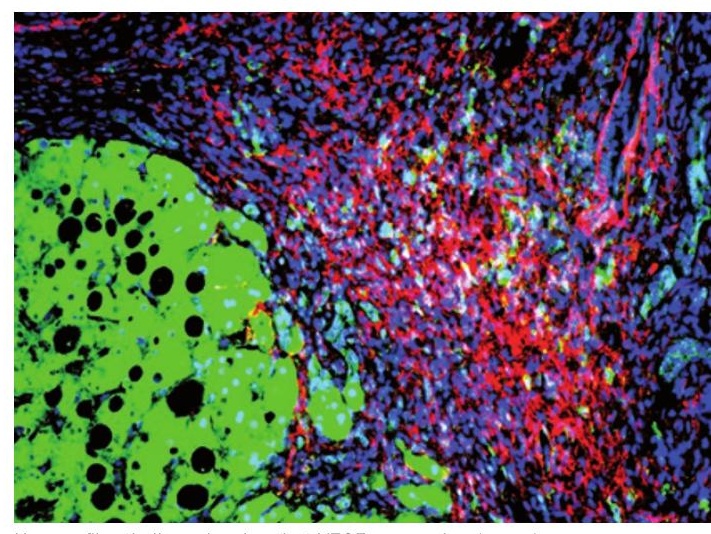

Human fibrotic liver showing that VEGF expression (green) is rather low. Image courtesy of C. Stockmann.

our findings, we will investigate whether targeted delivery of VEGF to the fibrotic scar tissue by different approaches can enhance the resolution of liver fibrosis."

Isobel Leake

Original article Kantari-Mimoun, C. et al. Resolution of liver fibrosis requires myeloid cell-driven sinusoidal angiogenesis. Hepatology doi:10.1002/hep.27635 